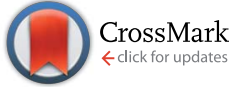

Cite this: RSC Adv., 2015, 5, 30067

Received 11th March 2015 Accepted 23rd March 2015

DOI: $10.1039 / \mathrm{c} 5 \mathrm{ra0} 4335 \mathrm{~b}$

www.rsc.org/advances

\section{Developmental and organ-specific toxicity of cucurbit[7]uril: in vivo study on zebrafish models $\uparrow$}

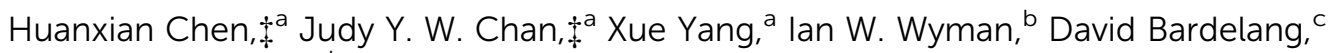 \\ Donal H. Macartney, ${ }^{b}$ Simon M. Y. Lee*a and Ruibing Wang*a
}

\section{Introduction}

Macrocyclic host molecules such as the cyclodextrins and calixarenes have received considerable attention as vehicles for drug delivery, due to their ability to improve the water solubility, bioavailability, physical and chemical stability, and release profiles of drugs. ${ }^{1-4}$ During recent years a new family of macrocyclic host molecules, the cucurbit $[n]$ urils $(\mathrm{CB}[n], n=5-8$, $10,14)$ have received increasingly intensive attention and have been the subject of a number of reviews due to their potential application in drug formulation and delivery. ${ }^{5,6} \mathrm{CB}[n]$ consists of $n$ glycoluril units that are bridged by $2 n$ methylene groups, forming a hydrophobic cavity and two identical carbonyl-lined portals with negative dipole potentials. The reaction of glycoluril and excess formaldehyde in the presence of concentrated sulfuric acid or hydrochloric acid at a temperature of $\sim 110{ }^{\circ} \mathrm{C}$ produces the macrocyclic $\mathrm{CB}[n]$ compounds through an acidcatalyzed condensation reaction, initially only $\mathrm{CB}[6]$ was isolated and characterized. ${ }^{7}$ In the years of 2000-2001, the research groups of Kim and Day independently isolated the homologues $\mathrm{CB}[5], \mathrm{CB}[7]$ and $\mathrm{CB}[8]$, using a lower temperature of $75-90{ }^{\circ} \mathrm{C}$ for the synthesis of these macrocycles. ${ }^{8,9}$ This development

${ }^{a}$ State Key Laboratory of Quality Research in Chinese Medicine, Institute of Chinese Medical Sciences, University of Macau, Taipa, Macao, China. E-mail: rwang@umac. mo; simonlee@umac.mo; Tel: +853-8822-4689

${ }^{b}$ Department of Chemistry, Queen's University, Kingston, ON, K7L 3N6, Canada ${ }^{c}$ Aix-Marseille Université, CNRS, Institut de Chimie Radicalaire, UMR 7273, 13397 Marseille, France

$\dagger$ Electronic supplementary information (ESI) available. See DOI: $10.1039 / \mathrm{c} 5 \mathrm{ra} 04335 \mathrm{~b}$

\$ These two authors contributed equally to this work. dramatically expanded the versatility of cucurbit $[n]$ uril chemistry and has attracted more scientists to this field. During the past fifteen years, $\mathrm{CB}[n] \mathrm{s}$ have demonstrated outstanding molecular recognition properties and superior interactions with a wide range of neutral and positively charged molecules, especially those possessing imine or amine groups, many of which are bioactive and medically relevant compounds. ${ }^{5,6,10}$

Among the $\mathrm{CB}[n]$ family, $\mathrm{CB}[7]$ (shown in Fig. 1) has received perhaps the greatest attention as a potential drug delivery vehicle due to its superior water-solubility and compatible size with various organic and organometallic drug molecules. ${ }^{5,6}$ Examples of drugs which have been studied with $\mathrm{CB}[7]$ include: atenolol (a beta-blocker) ${ }_{1}^{11}$ pyrazinamide (a tuberculosis drug), ${ }^{12}$ platinum-based anti-cancer drugs such as cisplatin, ${ }^{13}$ prilocaine (a local anaesthetic), ${ }^{14}$ coumarin (an anticoagulant), ${ }^{15}$ ranitidine (used to treat ulcers), ${ }^{16}$ and vitamin $\mathrm{B}_{12}$ as well as coenzyme $\mathrm{B}_{12} \cdot{ }^{17}$ For instance, we have reported the encapsulation of ranitidine by $\mathrm{CB}[7]$, and this encapsulation protected the drug from thermal degradation and could potentially extend the shelf-life of this drug. ${ }^{16}$ Similarly, we studied the encapsulation of vitamin $\mathrm{B}_{12}$ and coenzyme $\mathrm{B}_{12}$ by $\mathrm{CB}[7]$, through binding to the 5,6-dimethylbenzimidazole $\alpha$-nucleotide base, and observed stabilization of the base-off forms of these compounds. ${ }^{17}$ Wheate and coworkers reported that various platinum-based anticancer complexes have increased thermal stability and reduced cytotoxicity upon complexation with $\mathrm{CB}[7] .^{18,19}$ As demonstrated by these and other examples, the number of pharmaceutically relevant applications of $\mathrm{CB}[7]$ is rapidly expanding. Therefore, it has become critical to clearly understand the intrinsic toxicity profile of $\mathrm{CB}[7]$ before its application in real-world drug formulations. 


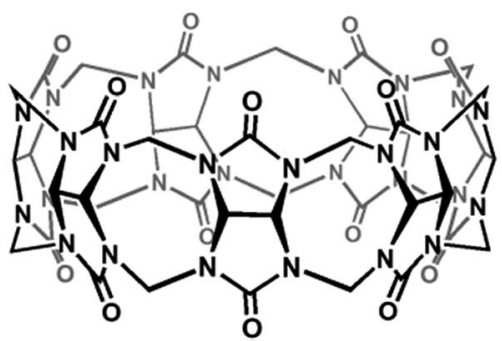

Fig. 1 The molecular structure of $\mathrm{CB}[7]$.

Several in vitro studies on cell cultures have shown that $\mathrm{CB}[7]$ exhibits very low toxicity at sub-mM concentrations or even up to $1 \mathrm{mM}$ concentrations. ${ }^{20,21}$ When $\mathrm{CB}[7]$ incubated with Chinese Hamster Ovary cells (CHKO-K1) for 2 days, the $\mathrm{IC}_{50}$ value was found to be $0.53 \mathrm{mM}$, and up to $1 \mathrm{mM}$ of $\mathrm{CB}[7]$ was found to be tolerable at shorter incubation times (e.g. $3 \mathrm{~h}) .{ }^{21}$ When assays were used to evaluate their metabolic activity and cytotoxicity (AK assay and MTS assay), $\mathrm{CB}[7]$ macrocycles have shown high cell tolerance at concentrations of up to $1 \mathrm{mM}$ in cell lines originating from the human kidney, liver or blood tissue. ${ }^{20}$ The effects observed for an intravenous single dose in vivo injection with a mice model demonstrated that $\mathrm{CB}[7]$ has a very low acute toxicity of $250 \mathrm{mg} \mathrm{kg}^{-1}$, based on a body weight change of less than $10 \%$ within 5 days of the injections. In addition, all of the mice injected via slow infusion into the vein began to recover after 5-8 days of the injections, thus the maximum tolerated dose (MTD) was established at approximately $250 \mathrm{mg} \mathrm{kg} \mathrm{kg}^{-1}$ dose level. ${ }^{21}$ A limitation of these in vitro and in vivo studies is that little information can be collected on the toxicity of $\mathrm{CB}[7]$ to specific organs. Very recently, the tissue specific toxicity including neuro-, myo- and cardiotoxicity of $\mathrm{CB}$ [7] has been examined with the use of ex vivo electrophysiological models. ${ }^{22}$ The study reported that $1 \mathrm{mM}$ of $\mathrm{CB}$ [7] did not exhibit statistically measurable neurotoxicity as examined using mouse sciatic nerve compound action potential. However, myotoxic and cardiotoxic activities were observed in the presence of $\mathrm{CB}[7]$ concentrations of $0.3 \mathrm{mM}$, which were determined by nerve-muscle force of contraction through chemical and electrical stimulation and changes in the rate and force of right and left atria contraction. ${ }^{22}$ Even with these very recent results, in vivo organ-specific toxicity profiles of $\mathrm{CB}[7]$ in live animal models are still not available. In addition, there has been no evaluation of $\mathrm{CB}[7]$ 's toxicity profile with regard to the development of any in vivo models.

Zebrafish has become a powerful and cost-efficient model for the evaluation of drug-induced toxicity, and it has been widely used for the assessment and prediction of developmental toxicity and organ-specific toxicities targeting the heart, central nervous system (CNS), the liver as well as the growth and development of living samples. ${ }^{23-28}$ One of the significant advantages of the zebrafish model is its rapid and synchronous growth. In addition, the transparency of the chorion and body enables non-invasive and direct observations during embryogenesis. More importantly, several reports have shown that the biological responses of zebrafish to small molecules and drugs are similar to that of mammals. ${ }^{29}$ In addition, the gene networks of zebrafish regulating embryonic development, normal physiology and pathogenesis also showed high conservation in comparison with humans. These features suggest that zebrafish shall provide an useful model for the evaluation of $\mathrm{CB}[7]$ toxicity that exhibits a safety profile that may be similar to that of humans. $^{30}$ With regard to studies of organ-specific toxicity, zebrafish has been identified as an ideal model for investigations involving cardiac function and human heart diseases. ${ }^{31}$ Functional and behavioral studies focusing on parameters such as hearing, aggression, learning and locomotion have also been developed that are useful for identifying neurotoxins without obvious phenotypic changes. ${ }^{32}$ The clear chorion and embryo of the zebrafish allow for continuous visualization of the anatomical changes associated with the development of this species. This visual clarity, along with short maturation times and the capability of complex behavior, makes this model particularly useful for measuring changes to the development phases. ${ }^{33}$ Meanwhile, zebrafish models have also been employed for hepatotoxicity studies to provide first-hand in vivo liver-specific toxicity data, although their liver anatomy differed from that of rodents and humans. ${ }^{34}$

Herein we will report our efforts to utilize zebrafish models to study the developmental and organ-specific toxicity profile of $\mathrm{CB}[7]$. The results obtained through these efforts will provide valuable insight into the toxicity of $\mathrm{CB}[7]$, which will be of immense importance with respect to the future use of $\mathrm{CB}[7]$ in the biomedical field.

\section{Materials \& methods}

\subsection{Zebrafish husbandry and breeding}

Wild type zebrafish were used for developmental toxicity, hepatotoxicity and locomotion and behavioral assays, while $\mathrm{Tg}$ (cmcl2:GFP) zebrafish with GFP (green fluorescent protein) specifically expressed in the myocardial cells were used for cardiotoxicity assays. ${ }^{35}$ The procedures for zebrafish culture, breeding, embryo collection, embryonic and larval culture, fluorescent observation were performed according to the standard procedures. ${ }^{36}$ Briefly, adult fish were raised in an aquaculture system with $12 \mathrm{~h}$ alternating light/dark cycles, and fed twice a day with newly hatched brine shrimp. Mature male and female zebrafish (at a ratio of 1 male to 2 females) were transferred into a breeding tank and separated by a mesh screen the night before breeding. All fertilized embryos were examined under a microscope and those that developed normally were selected for subsequent experiments. The collection of embryos, and exposure experiments were all performed in E3 medium (5 mM NaCl, $0.17 \mathrm{mM} \mathrm{KCl,} 0.33 \mathrm{mM} \mathrm{CaCl}_{2}, 0.33 \mathrm{mM}$ $\mathrm{MgSO}_{4}$ at $\mathrm{pH}$ 7.2-7.3). Zebrafish and embryos were maintained at $27 \pm 1{ }^{\circ} \mathrm{C}$. All experiments involving animals were conducted according to the ethical guidelines of the Institute of Chinese Medical Sciences, University of Macau.

\subsection{Developmental toxicity assay}

In order to determine the developmental toxicity of $\mathrm{CB}$ [7], the hatchability, morphology, survival rate and body length of the 
zebrafish were measured. During the hatchability study, at 1 day post fertilization (dpf), zebrafish embryos at the same developmental stage were randomly distributed into a 24-well microplate (14 to 17 fish per well) and exposed to $1 \mathrm{~mL}$ solutions of $\mathrm{CB}$ [7] at various concentrations $(0,125,250,500$, and $750 \mu \mathrm{M}$, respectively). After 2 days of incubation, the hatched larvae were counted in each group, and the hatchability was analyzed by the ratio of hatched numbers/total treated numbers $\times 100 \%$. In order to perform the morphology, survival rate and body length measurements, 1 dpf zebrafish embryos were dechorionated under microscope by the manual removal of the chorion with fine forceps, and then randomly distributed into a 24-well microplate (12 to 14 fish per well), before they were incubated with $1 \mathrm{~mL}$ solutions of $\mathrm{CB}[7]$ (at $0,125,250,500,750 \mu \mathrm{M}$, respectively) for 2 days. Survival rates were calculated, while the morphologies and body lengths of the zebrafish were measured using a Olympus DSU (Disk Scanning Unit) Confocal Imaging System.

\subsection{Cardiotoxicity assay}

$\mathrm{Tg}(\mathrm{cmlc2}$ :GFP) zebrafish embryos were used as cardiotoxicity assay. All embryos were cultivated in embryo medium containing $0.003 \%$ (wt\%) of 1-phenyl-2-thiourea (PTU) to block pigmentation since $1 \mathrm{dpf}$. Meanwhile, $2 \mathrm{dpf}$ zebrafish embryos were dechorionated as mentioned above, and then randomly distributed into a 24-well microplate (12 to 16 fish per well), treated with $1 \mathrm{~mL}$ solutions of $\mathrm{CB}[7]$ (at various concentrations including $0,125,250,500,750 \mu \mathrm{M}$ ) and with a $1 \mathrm{~mL}$ solution of doxorubicin $(40 \mu \mathrm{M}$, an anticancer drug with well-known cardiotoxicity) as a positive control. After 2 days of incubation, zebrafish were immersed into $1 \%$ (wt\%) low-melting point agarose matrix (Gibco) to fix them in a dorsal orientation and restrict their movement. The heart morphology, heart rates (HR) and quantitative assessment of other cardiac functions were obtained from $15 \mathrm{~s}$ video segments recorded of individual fish using an Olympus Cell^ $\mathrm{R}$ imaging system comprising IX71 microscope at room temperature. Ventricular functions were evaluated with various parameters, which were measured as described previously. ${ }^{37}$ Images from the video were used to measure the longitudinal axis length $(a)$ and lateral axis length (b) between the myocardial borders of ventricles at end-diastole and end-systole, respectively. In order to measure the $\mathrm{HR}$, the number of heartbeats in a $15 \mathrm{~s}$ interval was counted. The ventricular volume at end-diastole (EDV) and end-systole (ESV) in the larvae was calculated from the heart dimensions using the formula for a prolate spheroid: $V=4 / 3 \pi a b^{2}$. The stroke volume (SV), cardiac output (CO) and percent fractional shortening (\% FS) were calculated as follows: SV = (EDV - ESV), $\mathrm{CO}=\mathrm{SV} \times \mathrm{HR}, \mathrm{FS} \%=($ diastolic diameter - systolic diameter $) /$ (systolic diameter) $\times 100 \%$.

\subsection{Assessment of liver toxicity}

Zebrafish (4 dpf) were treated with $0,125,250,500,750 \mu \mathrm{M}$ of $\mathrm{CB}$ [7] in fish medium for 1 day. A fish medium sample that did not contain $\mathrm{CB}[7]$ was used as the blank control. Meanwhile, a fish medium sample containing $25 \mu \mathrm{M}$ amiodarone (ADR, with known liver toxicity) was used as the positive control. At the end of the treatment, 8-10 zebrafish from each group were randomly selected to be photographed under a microscope. The fish were anesthetized with $0.004 \%$ tricaine before they were placed dorso-laterally under the imaging system. The entire liver and yolk sac of each fish was measured, and analysis of the liver and yolk sac volumes were performed using the ImageJ software package.

\subsection{Assessment of locomotion and behavioral toxicity}

Zebrafish larvae (6 dpf) were placed into the wells of a 96-well plate (1 larvae per well). The fish was acclimatized for $30 \mathrm{~min}$ and their basal swimming behavior was monitored for $10 \mathrm{~min}$ using a Viewpoint Zebrabox system. Various concentrations of $\mathrm{CB}[7](0,125,250,500,750 \mu \mathrm{M})$ or $10 \mathrm{mM}$ pentylenetetrazol (PTZ, a chemical known to induce seizure) as a positive control were added to each well (8-10 fish in each group). The swimming behavior of the larvae was then recorded for $10 \mathrm{~min}$ starting at time point of $0 \mathrm{~min}, 15 \mathrm{~min}, 30 \mathrm{~min}, 60 \mathrm{~min}, 120 \mathrm{~min}$ and $240 \mathrm{~min}$, respectively. The swimming distance reached by each zebrafish was then analyzed using the Zebrabox system.

\section{Results and discussion}

\subsection{Developmental toxicity}

As shown in Fig. 2A, the mortality of the zebrafish increased when they were exposed to elevated concentrations of $\mathrm{CB}$ [7] for 2 days. No significant effect on the survival rate was observed for the 125 and $250 \mu \mathrm{M}$ treatment groups. However, one treatment of groups of zebrafish larvae ( $n=13-14$ in each group) with 500 and $750 \mu \mathrm{M}$ of $\mathrm{CB}[7]$ for 2 days resulted in $77 \%$ and $38 \%$ survival rates, respectively. None of the zebrafish survived when they were exposed to $\mathrm{CB}[7]$ at concentrations of $1 \mathrm{mM}$ or higher for 2 days. Thus the lethal dose $\mathrm{LD}_{50}$ is likely around $750 \mu \mathrm{M}$ for these $1 \mathrm{dpf}$ zebrafish larvae. With shorter incubation times such as within $24 \mathrm{~h}$, no mortality was observed even among the $750 \mu \mathrm{M} \mathrm{CB}[7]$ group, which indicates that extended exposure to $\mathrm{CB}[7]$ causes fish death. Similarly, the hatchability of zebrafish embryos also decreased in a dosage-dependent manner after the embryos had been exposed to $\mathrm{CB}[7]$ for 2 days. While exposure to $250 \mu \mathrm{M} \mathrm{CB}[7]$ showed moderate impact on the hatchability, higher concentrations of $\mathrm{CB}[7](>500 \mu \mathrm{M})$ significantly inhibited the hatchability of the zebrafish embryos when compared with that of the control group (Fig. 2B).

When the morphologies of these $\mathrm{CB}[7]$ treated fish were examined, no obvious morphological abnormalities, such as crooked bodies (CB), spinal deformities (SD), yolk sac edemas (YSE) or pericardial edema (PE), were observed (Fig. 3 top). CB [7] treatment did not exhibit any significant effects on the growth of the body length of zebrafish after 2 full days of incubation of $1 \mathrm{dpf}$ zebrafish. It is noteworthy that, even for the treatment group with $750 \mu \mathrm{M} \mathrm{CB}[7]$ that has shown high mortality, the fish that did survive still had similar body lengths with those of the control group, indicating that the physical growth of zebrafish larvae had a high tolerance to $\mathrm{CB}$ [7] (Fig. 3 bottom). Overall, these results suggested that $\mathrm{CB}[7]$ exhibited 
(A)

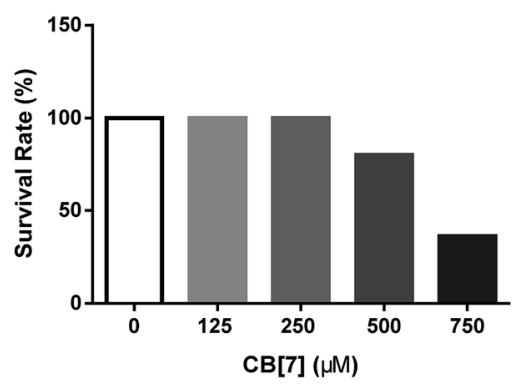

(B)

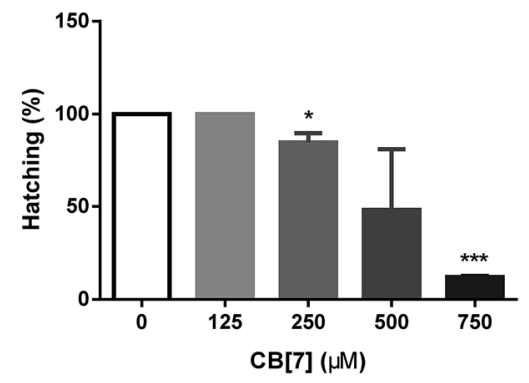

Fig. 2 Survival rates of zebrafish (1 dpf) exposed with various concentrations of CB[7] for 2 days (A), and hatchability of zebrafish embryos within 2 days of exposure to various concentrations of CB[7] (B). Data are presented as mean \pm S.D. $(n=14-17)$. $* P<0.05, * * * P<0.001$ significantly different compared with the control group.

certain toxic effects on the development (e.g. hatchability and mortality) of the zebrafish. At the same time, since no obvious morphological abnormalities or changes in the body length were observed for those fish that survived these mortality studies, we postulated that the developmental toxicity may be a non-lethal factor, and likely $\mathrm{CB}[7]$ toxicity on other organs or tissues may be the cause of zebrafish death.

\subsection{Cardiotoxicity}

To evaluate the cardiotoxicity of $\mathrm{CB}[7]$, cardiac morphology was evaluated first when the zebrafish were treated with various concentrations of $\mathrm{CB}[7]$. As shown in Fig. 4, representative images of zebrafish hearts displayed the systolic and diastolic functions after doxorubicin (Dox) or $\mathrm{CB}[7]$ treatment. Doxinduced cardiotoxicity has been widely reported both in humans and among laboratory animals, including mice, rats and zebrafish. ${ }^{38}$ Therefore, Dox was used as a positive control in our study. Exposure to Dox caused the hearts of the zebrafish to become distorted into an elongated shape with a thin atrium, a compact ventricle, and a massive pericardial edema. These morphological defects are related to cardiac dysfunction that has been well known. ${ }^{38}$ Exposure to the 125, 250 and $500 \mu \mathrm{M} \mathrm{CB}$ [7] solutions did not exhibit an obvious influence on the heart morphology. However, it seems that exposure to $750 \mu \mathrm{M} \mathrm{CB}[7]$ resulted in similar but less severe responses to those induced by Dox, but no pericardial edema was found. As expected, no signs of heart failure or edema were found among the zebrafish hearts in the control group.

Additionally, cardiac function was evaluated according to several parameters, including the stoke volume (SV), heart rate (HR), cardiac output (CO), and fractional shortening (FS). As demonstrated by Fig. 5, exposure to $500 \mu \mathrm{M}$ or higher concentrations of $\mathrm{CB}[7]$ dramatically decreased the SV, $\mathrm{CO}$, and FS, in comparison with the control group. Similar cardiotoxic effects on the SV, CO, and FS were observed upon exposure to Dox. Meanwhile, exposure to 125 and $250 \mu \mathrm{M} \mathrm{CB}$ [7] did not cause significant effects on these parameters. On the other hand, it is evident that Dox caused a moderate reduction of the HR. In contrast, $\mathrm{CB}[7]$ treatment slightly elevated the $\mathrm{HR}$ in a dosedependent manner in the concentration range of 125-750 $\mu \mathrm{M}$ (Fig. 5B). The cardiotoxicity profile of $\mathrm{CB}[7]$ seems to be

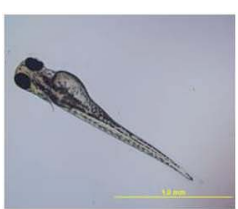

Ctrl

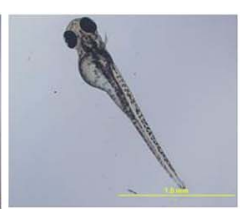

125

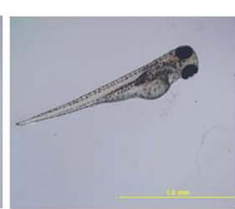

250

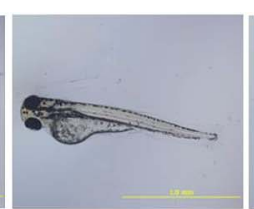

500

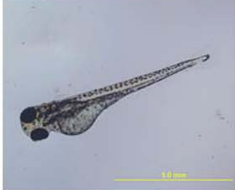

750

$\mathrm{CB}[7](\mu \mathrm{M})$

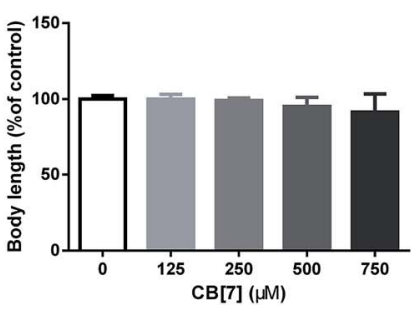

Fig. 3 Morphology of representative zebrafish larvae (3 dpf) after exposure to various concentrations of CB[7] for 2 days (top, scale bar: $1.0 \mathrm{~mm}$ ), and their body lengths of each group, relative to the control group, by the end of incubation (bottom). Data are presented as mean \pm S.D. ( $n=12$ ). No statistical difference was observed across these groups. 


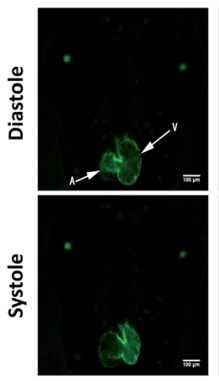

Ctrl

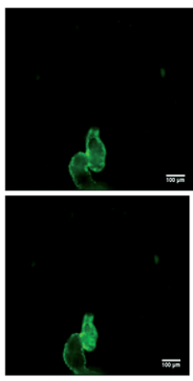

Dox $(40 \mu \mathrm{M})$

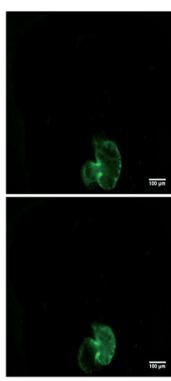

125

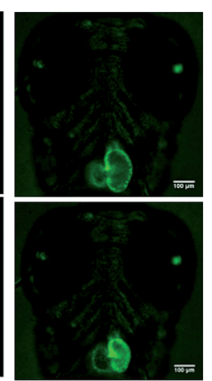

250

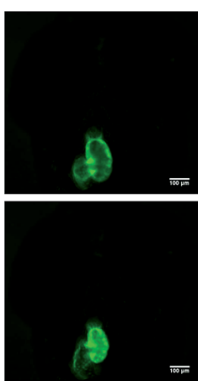

500

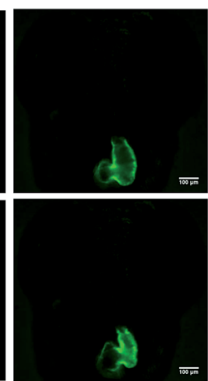

750

$\mathrm{CB}[7](\mu \mathrm{M})$

Fig. 4 Representative fluorescent microscopic images of Tg(cmcl2:GFP) zebrafish (4 dpf) with GFP specifically expressed in the myocardial cells, exposed to various concentrations of $\mathrm{CB}[7]$ or $40 \mu \mathrm{M}$ doxorubicin (Dox) as a positive control, for 2 days. "A" denotes atrium, and " $\mathrm{V}$ " denotes ventricle (Scale bar, 100 micron).

consistent with a observations reported in a recent ex vivo study. ${ }^{22}$

With these results in hand, we speculate that $\mathrm{CB}[7]$-induced heart failure may be a result of tachyarrhythmia or structural heart damage. It is generally accepted that tachycardia begets cardiac dysfunction and cardiac dysfunction begets tachycardia. ${ }^{39}$ As shown in Fig. 5B, tachycardia was observed in all treatment groups. However, significant reduction in the SV, CO and FS only appeared upon exposure to higher concentrations $(\geq 500 \mu \mathrm{M})$ of $\mathrm{CB}[7]$. This may be attributed to the initial compensatory mechanism, resulting in near normal stroke volume, cardiac output and fractional shortening. In more severe forms, greater increases in the HR can cause these parameters to deteriorate further and cause already reduced systolic function to worsen. ${ }^{40}$

\subsection{Locomotion and behavioral toxicity}

The locomotion and behavior, namely the total swimming distance (including short route and long route), was measured at several time points within a period of $4 \mathrm{~h}$ after the fish had been initially treated with various concentrations of $\mathrm{CB}[7]$ and $10 \mathrm{mM}$ PTZ (positive control). Exposure to PTZ, which is a convulsant and was thus used as the positive control here, caused a significant increase in the swimming distance at 15 min post treatment (Fig. 6B), but the fish recovered to normal swimming behaviors with longer time exposure. In contrast, it can be seen that $\mathrm{CB}[7]$ treatment significantly reduced the total swimming distance (in other words, inhibited the locomotion) of zebrafish after $2 \mathrm{~h}$ and $4 \mathrm{~h}$ of incubation in a dose-dependent manner at the concentration range of $250-750 \mu \mathrm{M}$ (Fig. 6E and F). In contrast, shorter incubation times with $\mathrm{CB}[7]$ did not
(A)

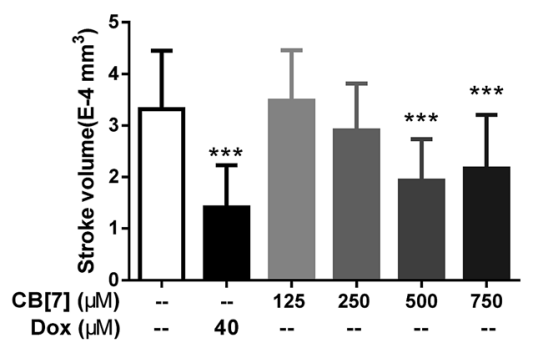

(C)

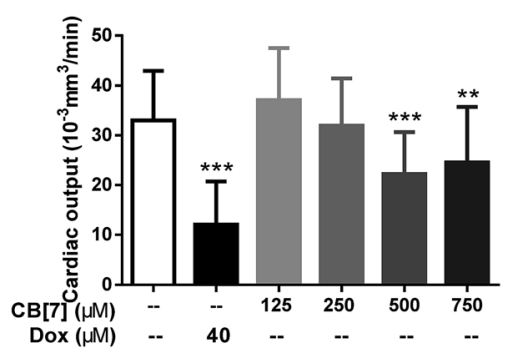

(B)

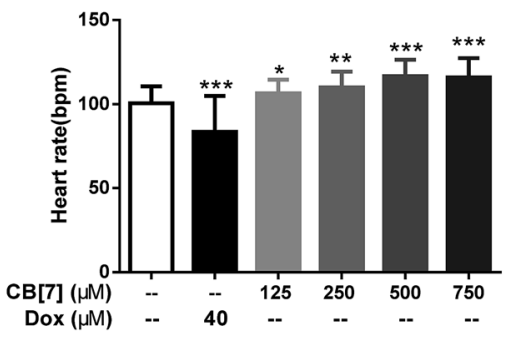

(D)

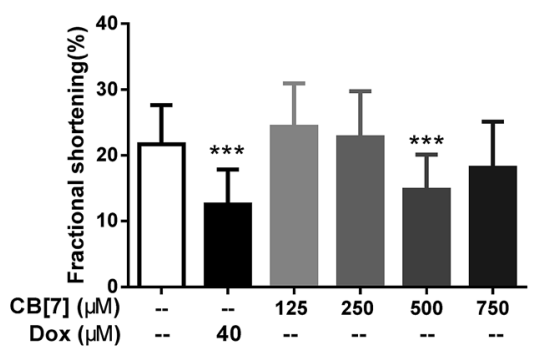

Fig. 5 Cardiac functions of zebrafish (2 dpf) exposed to various concentrations of CB[7] for 2 days. Stroke volume (A), heart rate (B), cardiac output (C) and \% FS (D) of zebrafish were shown. Data are presented as mean \pm S.D. $(n=16) . * P<0.05, * * P<0.01, * * * P<0.001$ significantly different compared with the control group. 
(A)

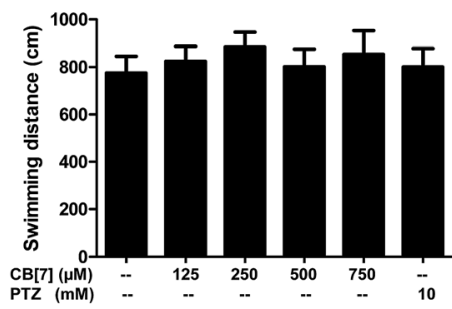

(D)

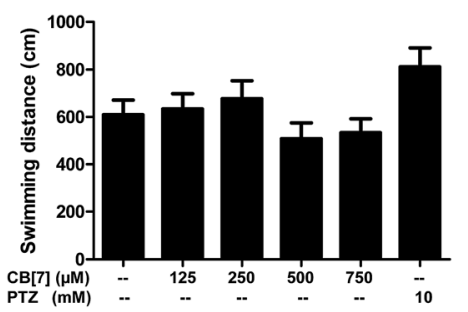

(B)

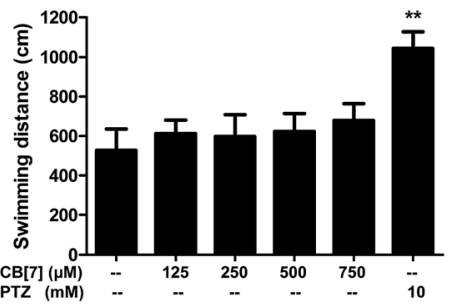

(E)

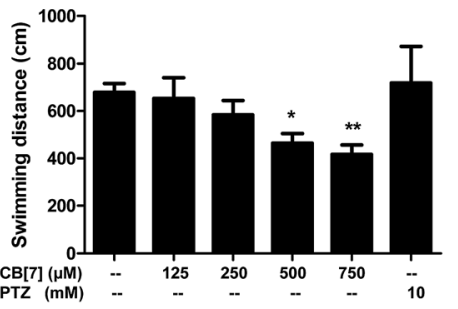

(C)

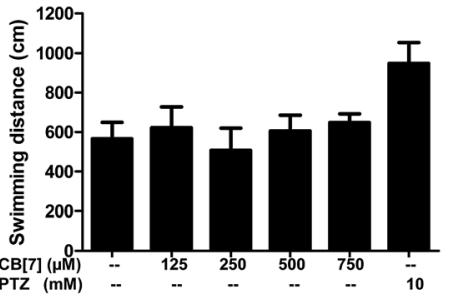

(F)

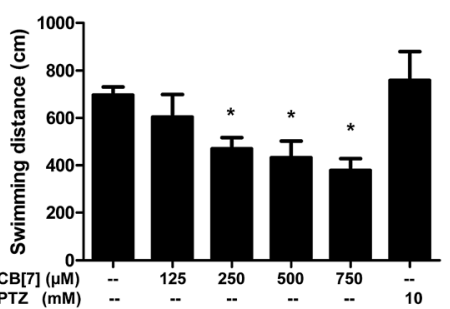

Fig. 6 Measurements of the total swimming distance of each fish in $10 \mathrm{~min}$ (wild type zebrafish at $6 \mathrm{dpf}$, the number of fish per group is 16 ) with CB[7] exposure durations of 0 (A), 15 (B), 30 (C), 60 (D), 120 (E), and 240 min (F). Data are presented as mean \pm S.D.\% $(n=8)$. *P<0.05, **P<0.01 significantly different compared with the control group.

result in statistically meaningful changes in the locomotion (see ESI $\uparrow$ for the recorded swimming patterns and paths).

In many studies, changes in locomotion have been regarded as a behavioral endpoint. ${ }^{41,42}$ The concept is simple but meaningful based on the idea that 'all behaviors are predicated on the ability to move'. ${ }^{42}$ Among investigations involving fish, locomotory activity endpoints include swimming speed, distance covered and turning rate. Associations between changes in zebrafish locomotions and neurotoxicity have also been noted reports investigating the mechanisms of addiction to amphetamines,${ }^{43}$ cocaine ${ }^{44}$ and ethanol ${ }^{45}$ Admittedly, non-neurological changes can also affect behaviour. For example, musculoskeletal injury and developmental abnormalities may be associated with behaviors that deviate from those exhibited by healthy individuals. However, in our study, the changes in the swimming behavior appeared within a short time of exposure $(2-4 \mathrm{~h})$ and no physical injuries or abnormalities were observed during subsequent physical examinations of the fish. Thus, we believe that the effect of $\mathrm{CB}[7]$ is likely on the neurological system, rather than the musculoskeletal system. In addition, the locomotion and behavior toxicity could be induced by other organ toxicity such as the already-seen cardiotoxicity. It is noteworthy
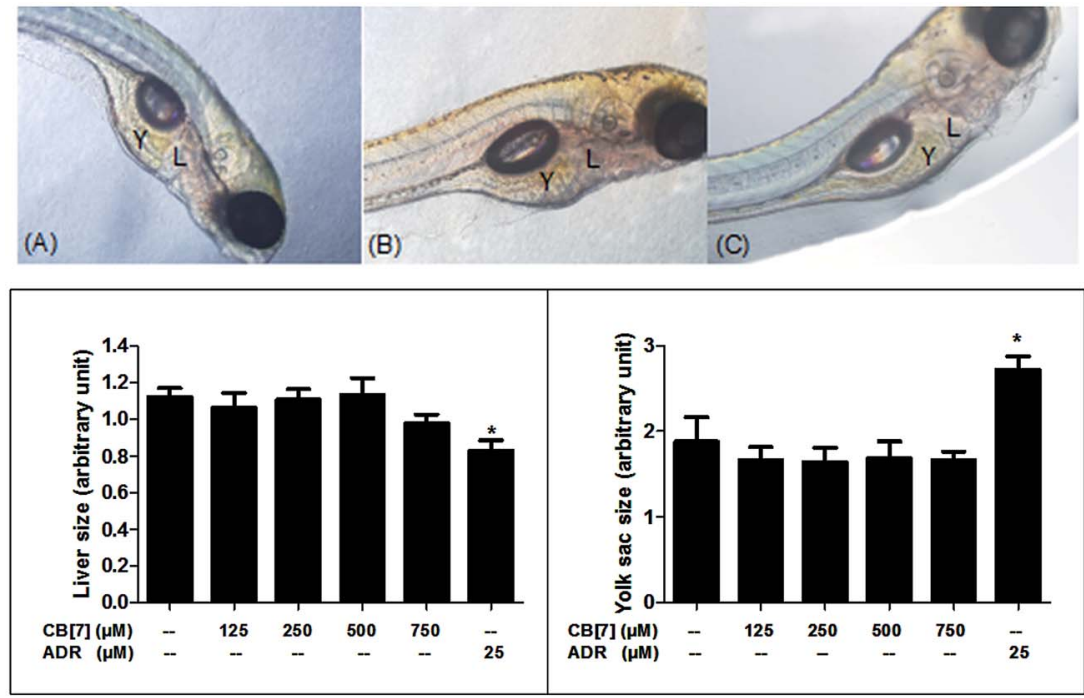

Fig. 7 Top panel: representative pictures showing the liver (L) and yolk sac (Y) of zebrafish larvae. (A) Negative control: zebrafish without treatment; (B) positive control: zebrafish treated with ADR $(25 \mu \mathrm{M})$ for $24 \mathrm{~h}$; (C) zebrafish treated with $750 \mu \mathrm{M}$ of CB[7] for $24 \mathrm{~h}$. Bottom panel: liver and yolk sac volume measurements of zebrafish treated with various concentrations of CB[7] or ADR ( $25 \mu \mathrm{M})$ for $24 \mathrm{~h}$. Data are presented as mean \pm S.D.\% $(n=8) . * P<0.05$ significantly different compared with the control group. 
that the previously reported ex vivo study did not find evidence of any measureable neurotoxicity arising from exposure to $1 \mathrm{mM}$ concentration $\mathrm{CB}[7]{ }^{22}$

\subsection{Hepatotoxicity}

Due to the similarity of the general structure, organization and functions of zebrafish liver to that of mammals, zebrafish has been used as a good vertebral model for drug metabolism studies. ${ }^{46}$ Another advantage of this model is that the liver of zebrafish is fully formed and functioning by $3 \mathrm{dpf}$, In our study, hepatotoxicity of $\mathrm{CB}[7]$ was determined by examining liver degeneration and yolk sac retention. As seen in Fig. 7, there were no obvious changes in either the liver size or the yolk sac size when the treated groups were compared with the control group. In contrast, exposure to $\operatorname{ADR}(25 \mu \mathrm{M}$, as a positive control) significantly decreased the liver size (liver degeneration) and induced yolk sac retention. Thus, the results demonstrated the non-toxic property of $\mathrm{CB}[7]$ itself and likely its metabolites as well. However, a limitation of this assay is that only phenotypic changes were observed. The change of hepatic enzyme activity, which is an important indicator of liver functions, could not be detected due to the limited size of the liver in zebrafish larvae. Nonetheless, the zebrafish liver's high tolerance to $\mathrm{CB}[7]$ at this concentration level seems to be supported by findings from previous investigations that utilized in vitro assays to evaluate the cytotoxicity of $\mathrm{CB}$ [7] (at concentrations of up to $1 \mathrm{mM}$ ) to cell lines originating from human liver tissue. ${ }^{20}$

\section{Conclusion}

In this study, the $\mathrm{CB}[7]$ was examined for its potential developmental and organ-specific toxicities in live in vivo zebrafish models for the first time. The results show that $\mathrm{CB}[7]$ exhibits measureable cardiotoxicity and locomotion and behavioral toxicity (likely a result of neurotoxicity) at concentrations of $0.50 \mathrm{mM}$ or higher, without significantly observable change on hepatotoxicity phenotype at concentrations up to $0.75 \mathrm{mM}$. In addition, $\mathrm{CB}$ [7] presented a $\mathrm{LD}_{50}$ likely around $0.75 \mathrm{mM}$ for 1 dpf zebrafish larvae when treated with $\mathrm{CB}$ [7] for 2 days, although interestingly at this concentration range $\mathrm{CB}[7]$ did not seem to affect the physical growth and morphology of the surviving fish. The functional concentration of $\mathrm{CB}$ [7] employed in drug complexation and delivery applications is often at $0.25 \mathrm{mM}$ or lower, and thus the overall in vivo toxicity profile is still safe at this concentration range. Consequently, these results still support future exploration of the use of $\mathrm{CB}[7]$ in pharmaceutical sciences and medicinal chemistry at sub-toxic concentrations, and these results warrant further investigation of the biosafety of $\mathrm{CB}[7]$ in large animal models and human tissue samples.

\section{Acknowledgements}

We wish to thank the University of Macau (Project no. SRG201400025) and NSERC-Canada for financial support of this project.

\section{References}

1 M. E. Brewster and T. Loftsson, Adv. Drug Delivery Rev., 2007, 59, 645-666.

2 T. Loftsson, S. B. Vogensen, M. E. Brewster and F. Konradsdottir, J. Pharm. Sci., 2007, 96, 2532-2546.

3 T. Loftsson and M. E. Brewster, J. Pharm. Pharmacol., 2010, 62, 1607-1621.

4 E. V. Ukhatskaya, S. V. Kurkov, S. E. Matthews and T. Loftsson, J. Pharm. Sci., 2013, 102, 3485-3512.

5 D. H. Macartney, Isr. J. Chem., 2011, 51, 600-615.

6 S. Walker, R. Oun, F. J. McInnes and N. J. Wheate, Isr. J. Chem., 2011, 51, 616-624.

7 W. A. Freeman, W. L. Mock and N. Y. Shih, J. Am. Chem. Soc., 1981, 103, 7367-7368.

8 J. Kim, I. S. Jung, S. Y. Kim, E. Lee, J. K. Kang, S. Sakamoto, K. Yamaguchi and K. Kim, J. Am. Chem. Soc., 2000, 122, 540541.

9 A. Day, A. P. Arnold, R. J. Blanch and B. Snushall, J. Org. Chem., 2001, 66, 8094-8100.

10 K. I. Assaf and W. M. Nau, Chem. Soc. Rev., 2015, 44, 394-418. 11 F. J. McInnes, N. G. Anthony, A. R. Kennedy and N. J. Wheate, Org. Biomol. Chem., 2010, 8, 765-773.

12 N. J. Wheate, V. Vora, N. G. Anthony and F. J. McInnes, J. Inclusion Phenom. Macrocyclic Chem., 2010, 68, 359-367.

13 N. J. Wheate, D. P. Buck, A. I. Day and J. G. Collins, Dalton Trans., 2006, 451-458.

14 I. W. Wyman and D. H. Macartney, Org. Biomol. Chem., 2010, 8, 247-252.

15 R. B. Wang, D. Bardelang, M. Waite, K. A. Udachin, D. M. Leek, K. Yu, C. I. Ratcliffe and J. A. Ripmeester, Org. Biomol. Chem., 2009, 7, 2435-2439.

16 R. B. Wang and D. H. Macartney, Org. Biomol. Chem., 2008, 6, 1955-1960.

17 R. B. Wang, B. C. MacGillivray and D. H. Macartney, Dalton Trans., 2009, 3584-3589.

18 A. R. Kennedy, A. J. Florence, F. J. McInnes and N. J. Wheate, Dalton Trans., 2009, 7695-7700.

19 N. J. Wheate, J. Inorg. Biochem., 2008, 102, 2060-2066.

20 G. Hettiarachchi, D. Hguyen, J. Wu, D. Lucas, D. Ma, L. Isaacs and V. Briken, PLoS One, 2010, 5, e105014.

21 V. D. Uzunova, C. Cullinane, K. Brix, W. M. Nau and A. I. Day, Org. Biomol. Chem., 2010, 8, 2037-2042.

22 R. Oun, R. S. Floriano, L. Isaacs, E. G. Rowan and N. J. Wheate, Toxicol. Res., 2014, 3, 447-455.

23 P. McGrath and C. Q. Li, Drug Discovery Today, 2008, 13, 394401.

24 T. P. Barros, W. K. Alderton, H. M. Reynolds, A. G. Roach and S. Berghmans, Br. J. Pharmacol., 2008, 154, 1400-1413.

25 (a) C. B. Lovely, R. D. Nobles and J. K. Eberhart, Alcohol, 2014, 48, 595-602; (b) L. Huang, Z. Zuo, Y. Zhang and C. Wang, Aquat. Toxicol., 2015, 158, 157-164; (c) Y. Jin, Z. Liu, T. Peng and Z. Fu, Fish Shellfish Immunol., 2015, 43, 405-414; (d) S. Zhuang, Z. Zhang, W. Zhang, L. Bao, C. Xu and H. Zhang, Aquat. Toxicol., 2015, 159, 119-126. 
26 S. H. Lam, C. Y. Ung, M. M. Hlaing, J. Hu, Z. H. Li, S. Mathavan and Z. Y. Gong, Biochim. Biophys. Acta, Gen. Subj., 2013, 1830, 4778-4789.

27 H. S. Jones, H. T. Trollope, T. H. Hutchinson, G. H. Panter and J. K. Chipman, Xenobiotica, 2012, 42, 1069-1075.

28 A. A. Amali, R. D. Rekha, C. J. F. Lin, W. L. Wang, H. Y. Gong, G. M. Her and J. L. Wu, J. Biomed. Sci., 2006, 13, 225-232.

29 (a) A. J. Hill, H. Teraoka, W. Heideman and R. E. Peterson, Toxicol. Sci., 2005, 86, 6-19; (b) R. T. Peterson, R. Nass, W. A. Boyd, J. H. Freedman, K. Dong and T. Narahashi, Neurotoxicology, 2008, 29, 546-555.

30 (a) K. Sheehan-Rooney, B. Palinkasova, J. K. Eberhart and M. J. Dixon, Dev. Dyn., 2010, 239, 3481-3491; (b) M. E. Swartz, K. Sheehan-Rooney, M. J. Dixon and J. K. Eberhart, Dev. Dyn., 2011, 240, 2204-2220.

31 (a) W. Heideman, D. S. Antkiewicz, S. A. Carney and R. E. Peterson, Cardiovasc. Toxicol., 2005, 5, 203-214; (b) J. Chen, Zebrafish, 2013, 10, 389-400; (c) J. Bakkers, Cardiovasc. Res., 2011, 91, 279-288; (d) C. C. Huang, A. Monte, J. M. Cook, M. S. Kabir and K. P. Peterson, Assay Drug Dev. Technol., 2013, 11, 561-572; (e) Y. H. Chen, C. W. Pai, S. W. Huang, S. N. Chang, L. Y. Lin, F. T. Chiang, J. L. Lin, J. J. Hwang and C. T. Tsai, J. Am. Heart Assoc., 2013, 2, e000231.

32 (a) P. I. Bang, P. C. Yelick, J. J. Malicki and W. F. Sewell, J. Neurosci. Methods, 2002, 118, 177-187; (b) C. Parng, C. Ton, Y. X. Lin, N. M. Roy and P. McGrath, Neurotoxicol. Teratol., 2006, 28, 509-516.

33 (a) N. S. Sipes, S. Padilla and T. B. Knudsen, Birth Defects Res., Part C, 2011, 93, 256-267; (b) J. Bailey, A. Oliveri and E. D. Levin, Birth Defects Res., Part C, 2013, 99, 14-23.

34 (a) T. Tao, J. R. Peng and J. Genet, Genomics, 2009, 36, 325334; (b) R. Zhao and S. A. Duncan, Hepatology, 2005, 41, 956967.

35 C. J. Huang, C. T. Tu, C. D. Hsiao, F. J. Hsieh and H. J. Tsai, Dev. Dyn., 2003, 228, 30-40.
36 M. Westerfield, The Zebrafish Book. A Guide for the Laboratory Use of Zebrafish (Danio rerio), University of Oregon Press, 2000.

37 (a) Z. Chen, W. Huang, T. Dahme, W. Rottbauer, M. J. Ackerman and X. Xu, Cardiovasc. Res., 2008, 79, 97108; (b) M. Fink, C. Callol-Massot, A. Chu, P. Ruiz-Lozano, J. C. Izpisua Belmonte, W. Giles, R. Bodmer and K. Ocorr, BioTechniques, 2009, 46, 101-113; (c) R. Kopp, T. Schwerte and B. Pelster, J. Exp. Biol., 2005, 208, 2123-2134.

38 (a) A. L. Ferreira, L. S. Matsubara and B. B. Matsubara, Cardiovasc. Hematol. Agents Med. Chem., 2008, 6, 278-281; (b) H. Bar-Joseph, S. M. Stemmer, I. Tsarfaty, R. Shalgi and I. Ben-Aharon, J. Visualized Exp., 2015; (c) Y. Ichikawa, M. Ghanefar, M. Bayeva, R. Wu, A. Khechaduri, S. V. Naga Prasad, R. K. Mutharasan, T. J. Naik and H. Ardehali, J. Clin. Invest., 2014, 124, 617-630; (d) Z. Y. Zhou, L. L. Wan, Q. J. Yang, Y. L. Han, Y. Li, Q. Yu, C. Guo and X. Li, Toxicol. Appl. Pharmacol., 2013, 272, 238-244.

39 A. Bamimore and P. Mounsey, Heart Fail. Clin., 2013, 9, 501514.

40 D. H. MacIver, M. J. Dayer and A. J. Harrison, Int. J. Cardiol., 2013, 165, 25-34.

41 W. Boehmler, T. Carr, C. Thisse, B. Thisse, V. A. Canfield and R. Levenson, Genes, Brain Behav., 2007, 6, 155-166.

42 O. V. Anichtchik, J. Kaslin, N. Peitsaro, M. Scheinin and P. Panula, J. Neurochem., 2004, 88, 443-453.

43 T. D. Irons, R. C. MacPhail, D. L. Hunter and S. Padilla, Neurotoxicol. Teratol., 2010, 32, 84-90.

44 M. A. Lopez-Patino, L. Yu, H. Cabral and I. V. Zhdanova, Physiol. Behav., 2008, 93, 160-171.

45 R. Gerlai, M. Lahav, S. Guo and A. Rosenthal, Pharmacol., Biochem. Behav., 2000, 67, 773-782.

46 M. W. Hung, Z. J. Zhang, S. Li, B. Lei, S. Yuan, G. Z. Cui, P. M. Hoi, K. Chan and S. M. Y. Lee, J. Evidence-Based Complementary Altern. Med., 2012, 2012, 605303. 